International Journal of Wireless \& Mobile Networks (IJWMN) Vol. 7, No. 5, October 2015

\title{
INTEROPERABILITY AND QUALITY ASSURANCE FOR MULTI-VENDOR LTE NETWORK
}

\author{
Ramprasad Subramanian, Roshanak Heidari and Kumbesan Sandrasegaran \\ School of Computing and Communications, Centre for Real Time Information Networks, \\ Faculty of Engineering and Information Technology, University of Technology Sydney, \\ Sydney, Australia.
}

\begin{abstract}
The deployment of the LTE is picking up pace in many countries and these networks are deployed alongside the existing $2 G / 3 G$ services. LTE/LTE-A networks offer higher data rates and reduced delay to the subscribers. Today's mobile networks consist of equipment from multiple vendors and they are called multiple vendor networks. Interoperability testing is important at initial network launch and during network expansion. This paper discusses a typical problem related to interoperability testing along with the test results and the issues faced during the testing. The test results discussed in the paper are obtained from three scenarios - before testing, during testing and after testing. The test results are used to study the impact on network performance. Apart from the interoperability testing, an outline of testing that focus on general network stability, the interworking capability of LTE with other technologies such as $2 G$ and $3 G$ and taxonomy for the generation of key performance indicators (KPIs) are also discussed.
\end{abstract}

\section{KEYWORDS:}

KPIs, Interoperability, LTE, Testing.

\section{INTRODUCTION}

Long term evolution (LTE) is an evolution from the GSM/EDGE and WCDMA/HSPA and it was designed to provide higher data rates in economically viable way for mobile broadband services. The LTE system is based on SC-FDMA for uplink and OFDMA in the downlink side while the system architecture evolution (SAE) is a flat and all IP architecture with the separation for traffic in control and user planes. 3GPP has standardised both the approaches. Before the introduction of LTE, market analysis reported that data usage in WCDMA/HSPA and CDMA networks were growing rapidly and they expected the situation will improve after LTE is introduced. Many mobile operators planned for early deployments of LTE networks to cater for the subscribers demand. The first commercial LTE deployment was in the second half of 2010. To provide efficient, smooth and stable services to the subscribers, various tests were carried out in the lab and in the trial sites by both the operators and the vendors. These test results were used for benchmarking and helped the operators and vendors to compare with the live network results.

The LTE/SAE trial initiative (LSTI) (LSTI, 2008) is an open group founded by the various telecommunication industry leaders that comprised of vendors and operators who are working together to speed up the development of LTE/SAE. The initiative developed a proof of concept (POC) activity which consolidates the measurements from the leading telecom vendors to exhibit

DOI : 10.5121/ijwmn.2015.7505 
that the target performances of LTE are achievable. In addition to the peak data rates and minimal latency, the outcome reveals the results of the performance that the operators can offer to the subscribers. LSTI also focuses on the interoperability testing (IOT) between various vendors. Since the LTE deployment were carried out in urban and sub-urban initially, the equipment vendors who were contributing actively in the LSTI group deployed test sites in the major cities that simulated the exact conditions of the operators. These tests were carried with the $2 \times 2$ MIMO antenna configurations using slim-line cross-polarized multiband antennas that are used in $3 \mathrm{G}$ commercial deployments.

As the tradition, a wide range of key performance indicators (KPIs) were used by the mobile network operators and equipment vendors to analyse the network performance and quality to ensure that operator's targets are met. Maintenance of KPIs has always been a contentious issue between the operators and the equipment vendors. KPIs are always selected from the bottom up but often not coordinated properly, and this results in a ambiguous calculations of network performances. Sometimes, at the behest of the network operators, equipment vendors invest considerable energy and time to improve selected KPIs, but at the cost of other KPIs. KPI selection is another important activity for network operators. If KPIs are not selected properly, then it would have least impact in monitoring the network performance and they will not provide factual condition of the network. Network operators needs to be assured by the equipment vendors, that the KPIs defined for the network should reflect the tangible user behaviour and the user experience. For example, a KPI on LTE radio bearer can be dropped when analysing LTE device, since the device is not transmitting the data. These type KPIs does not offer precise understanding of the subscriber experience, since the sessions can be established quickly when required and delays goes unobserved by the subscribers.

The competition among the mobile network operators to attract more subscribers and the increasing intent of the people to subscribe to more data services with various QoS, are the two big catalysts to increase the number of $4 \mathrm{G}$ networks deployed in the market. The LTE networks have to be integrated with other traditional networks such as $2 \mathrm{G}$ and $3 \mathrm{G}$, thus creating an overlay on the existing networks. In some cases, the deployment can be a green field roll out. Given the operators past experiences and the challenges involved, in evolving the GSM network to UMTS, there is undoubtedly some apprehension in transforming the network to a flat all IP LTE network (Nokia, 2011). This apprehension made the majority of the operators to have the LTE network laid separately without any convergence to the existing network. Apart from the past experiences of the operators, technically LTE does not have any similarities with the $2 \mathrm{G}$ or $3 \mathrm{G}$ and it's a totally different kind of network. They have a different synchronization mechanism among the nodes. By introducing the LTE as a separate overlay, wireless operators have able to test the network without causing many disturbances to the existing networks such as $2 \mathrm{G}$ and $3 \mathrm{G}$. With the overlay decision, the operators are faced with another major decision on how to integrate the LTE with $2 \mathrm{G}$ and $3 \mathrm{G}$ seamlessly and without causing much trouble to the customers.

Competition among the mobile operators makes them to introduce new features in the network such as voice over LTE (VoLTE) and high definition video on demand (VoD). In order to keep abreast with the new features, various testing methods should be introduced and various metrics should be accurately measured so that the performance indicators of the networks are properly understood and corrected. To attain high quality of service, regular optimization of network is required and the implementation of self organizing networks (SON) becomes essential. This way of creating automation in the network helps mobile operators in reducing the operations cost of the network.

LTE networks are experiencing a huge surge in the amount of the signalling load and network events and this behaviour can be attributed to the increase of smart phones in the market. Therefore, the network performance measurements and the interpretation of the measurements 
should be understood properly and the operator's goals are met by maintaining the signalling load and providing the best QoS as much as possible to the subscribers.

Smartphone behaviour, adds complexity to the performance measurement. Generally, the traffic generated by these devices is different compared to the traffic generated by the USB dongle. The behaviour of the devices in the network changes with the new software upgrade, new model of devices introduced in the market and with the introduction of new apps, and this will affect the interpretation of KPIs. KPI management is difficult in a single technology environment. In a PLMN, which encompasses different flavours of technologies such as $2 \mathrm{G}, 3 \mathrm{G}$ and $4 \mathrm{G}$, the intensity of the complexities increases significantly and the resilience level of the KPIs as the true compilation of ground realities should be shored up.

At this juncture, an analogy is worth comparing here. The electrical standards of different countries are different and similarly, measurement of the network performances varies with the vendors. But as a single metric system, the KPI measurement and the definition by different vendors should be harmonised with the standards laid down by the standards institutes such as ITU and ETSI etc. In general, the KPI management framework should consist of metrics complying with the standards institutes such as ITU and ETSI. The parameter settings of the elements should comply with the standards laid down by these institutes, only the relevant KPIs related to the users experience should be included, the KPI measurement procedure should be explained adequately, KPI validation tests should be carried out to verify whether the defined KPIs meets the operators objective (Ericsson, 2011).

Handling of the spectrum is another major issue faced by the operators. Since frequencies are scarce resources and providing high data rates to the subscribers is very difficult without the management of these resource. Hence, a new technique of carrier aggregation (CA) is introduced in LTE-A. CA is one of the important feature of LTE-A that was standardised by 3GPP recently in Release- 10. This feature is designed to satisfy the IMT advanced requirements. In this feature, the eNodeB (eNB) can combine multiple spectrum bands to support high data rates in both uplink and downlink. Each carrier handles the traffics separately that are subdivided and then transmitted using physical layer resources of the carrier. This requires a separate link level mechanisms like hybrid automatic repeat request (HARQ) and control signalling for each carrier component. The backward compatibility of the LTE-A with LTE is very important. The LTE device that does not support LTE-A, will use one of the band and the handsets that support CA, will use multiple spectrum bands to send and receive data.

There are two types of CA configurations used in LTE-A and they are continuous CA and noncontinuous CA.

- Continuous CA

In this mode, the spectrum bands which contain $20 \mathrm{MHz}$ should be arranged adjacent so that they can be aggregated to create $40 \mathrm{MHz}$ band as a single spectrum.

\section{- Non-continuous CA}

In this mode, the aggregated carriers can be non-contiguous and can be from different bands e.g., an aggregation can be formed between two frequency bands such as $800 \mathrm{MHz}$ and $2.6 \mathrm{GHz}$ which are in different location of the spectrum bands. The channel characteristics such as path loss, building penetration loss and Doppler shift will have different behaviour in different frequency bands and these variations can be minimized in the scheduler through radio resource management (RRM). 


\section{TeST DESIGN METHODOLOGIES}

In general, testing can be defined as a process or methodology that is used to determine how well something works. The competition challenges the mobile network operators to increase the network capacity very rapidly by overlaying LTE network on top of $2 \mathrm{G}$ and $3 \mathrm{G}$ makes the networks complex in every aspect such as design and optimization and testing and verification. Therefore, an accurate method of testing a network is always important to analyse the performance of a network. In this section various test conditions for various modules of the LTE network have been discussed, to find out how well it works. The KPI verification strategies discussed in the forthcoming sections were put to practical tests and the results are plotted. The output of these results was discussed with the operators to check whether the strategy meets their expectations. The KPI testing led to discovery of an interoperability issue between SGW and MME between the two different vendors were identified and the issue was fixed.

\subsection{LTE network testing scenarios}

This section briefs about the various testing scenarios that needs to be tested to verify the performance of the network. The scenarios are classified as network stability, network behaviour in the cell edge, throughout analysis, scheduler behaviour analysis, modulation schemes to test the UEs and MIMO switching quantification. Each scenario are explained below in detail.

1. LTE network stability: The main purpose of this test is to find out how stable is the network. The performance of the radio links should be tested under the realistic scenarios, such as typical urban, suburban and rural environments. The test case should be performed with different traffic conditions to analyze the network stability.

2. Network behaviour in the cell edge: The purpose of this test is to demonstrate the performance of the network's data throughput rate for the subscribers located in the cell edge area and to select the antenna heights and near line of sight (LOS) propagations to enable the required throughput rate.

3. Throughput rate analysis: Through this test, the maximum achievable throughput for single user and multiple users in different environments such as indoors and outdoors can be analysed.

4. Scheduler behaviour analysis: The purpose of this test is to analyse the scheduler behaviour of the LTE base station for various QoS scenarios under varying channel environments.

5. Modulation schemes: The user equipment (UE) speeds should be tested under diverse modulation schemes can be tested in this test case.

6. MIMO switching mode quantification: Impact analysis should be made for adaptive MIMO switching modes with single data stream and with dual data stream in the real time network conditions.

\subsection{Testing of network KPIs}

The ITU-T along with the ETSI (ETSI, 2009) (3GPP, 2009) has prescribed the standardisations that needs to be adhered to provide better QoS for the subscribers. Adherence to these standards provides better QoS and provides excellent service accessibility, retainability, mobility and integrity. 


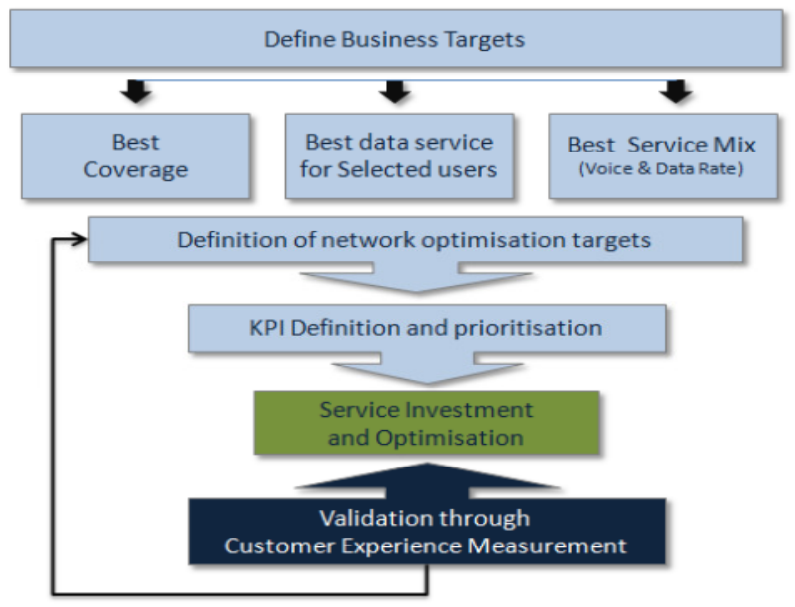

Fig. 1: ITU recommended KPI methodology

Figure 1 illustrates the KPI methodology that adheres to the ITU recommendation to measure subscriber experience and network quality. In general, the adeptness of a network can be demonstrated in a lab which will not have any semblance to the live network. These demonstrations are usually carried out with high end user terminals, with co-located evolved packet core (EPC) with eNB, with well defined interfaces etc. But this conditions does not exist in the real time network. In real time network, the performance and capacity goes in tandem. Both are very important parameters in a real time network. Hence, a trade-off between these parameters should be considered. These conditions have to be considered carefully while designing a test bed to design reliable KPIs to measure the user experience. Hence, the tests should be conducted using commercial available terminals, standard network settings, and transparent calculations of KPIs.

To implement the ITU recommended KPI methodology, a structured and planned approach is considered where many performance indices (PI) events are combined to create a few KPIs which can best indicate the user experience. Once a network becomes to operation mode, KPIs based on the counters should be used to monitor the performance of the network. These KPIs based on commercial traffic and network counters and are likely to differ from those selected for cluster tuning which are based on drive test measurements. Tuning and verification activities are designed to offer maximum benefit to the operator. Focused verification can be based on factors such as the location of key users, areas of dense traffic, difficult environments and known trouble spots.

\subsection{Clustering in LTE network}

Clustering, plays a vital role in large multi-networks to obtain scalability, reducing energy consumption and achieving better network performance. In clustering, the cluster heads $(\mathrm{CH})$ aggregates the data and reduces the traffic significantly. This model works in two ways 1) periodic selection of $\mathrm{CH}$ and 2) assignment of nodes to the clusters. A suitable strategy has to be employed to select the appropriate KPIs to analyse the clustering of the network. The vendors and operators should work in tandem on these KPIs and there should be a clear understanding between them about what KPIs are required and how it is designed before the actual start of the testing. In fact, it would be better if these types of agreements are reached before the start of official the contract. The Table 1 below summarize the broad KPI frame work commonly used KPIs design by the vendors. 
Table 1: KPI frame work

\begin{tabular}{|c|l|}
\hline CATEGORY & \multicolumn{1}{|c|}{ DESCRIPTION } \\
\hline Accessibility & $\begin{array}{l}\text { This type of KPIs gives } \\
\text { an idea about the } \\
\text { session setup and } \\
\text { session success rate in } \\
\text { the well defined } \\
\text { condition. }\end{array}$ \\
\hline $\begin{array}{c}\text { Service } \\
\text { retention }\end{array}$ & $\begin{array}{l}\text { This category gives an } \\
\text { idea about the service } \\
\text { continuity and it can be } \\
\text { used to calculate } \\
\text { whether abnormal } \\
\text { failures occurs such as } \\
\text { dropped calls. }\end{array}$ \\
\hline Service & $\begin{array}{l}\text { This will help to check } \\
\text { whether the obtained } \\
\text { service faces any } \\
\text { impairments because of } \\
\text { uplink or downlink } \\
\text { throughput or packet } \\
\text { loss. }\end{array}$ \\
\hline $\begin{array}{c}\text { Mobility } \\
\text { management }\end{array}$ & $\begin{array}{l}\text { This section covers } \\
\text { various handover types } \\
\text { both success and failure } \\
\text { conditions }\end{array}$ \\
\hline
\end{tabular}

Table 2: KPI framework for LTE network verification

\begin{tabular}{|l|l|l|l|}
\hline KPI & LAB & $\begin{array}{l}\text { SITE } \\
\text { ROLL } \\
\text { OUT }\end{array}$ & CLUSTER \\
\hline $\begin{array}{l}\text { Cell } \\
\text { availability }\end{array}$ & Yes & Yes \\
\hline $\begin{array}{l}\text { Session setup } \\
\text { success rate }\end{array}$ & Yes & Yes \\
\hline $\begin{array}{l}\text { Abnormal } \\
\text { session } \\
\text { release rate }\end{array}$ & Yes & Yes & Yes \\
\hline RTT & Yes & Yes & Yes \\
\hline $\begin{array}{l}\text { RTT packet } \\
\text { loss }\end{array}$ & Yes & & \\
\hline $\begin{array}{l}\text { Uplink } \\
\text { packet loss }\end{array}$ & Yes & & \\
\hline $\begin{array}{l}\text { Downlink } \\
\text { packet loss }\end{array}$ & & \\
\hline
\end{tabular}




\begin{tabular}{|l|l|l|l|}
$\begin{array}{l}\text { Throughout } \\
\text { (uplink and } \\
\text { downlink }\end{array}$ & PI & PI & PI \\
\hline $\begin{array}{l}\text { Handover } \\
\text { success rate }\end{array}$ & & Yes & Yes \\
\hline $\begin{array}{l}\text { Voice-session } \\
\text { setup time }\end{array}$ & Yes & & \\
\hline $\begin{array}{l}\text { Voice-session } \\
\text { setup success }\end{array}$ & Yes & & \\
\hline $\begin{array}{l}\text { Voice-session } \\
\text { abnormal } \\
\text { failure rate }\end{array}$ & Yes & & \\
\hline
\end{tabular}

The Table 2 typically summarizes the KPI framework for LTE network verification. In this table, each KPI must be tested under various conditions such as controlled environment, during site acceptance and during the service operation stage. The content of the table may vary depending on the operators. The table must be designed in consultation with the operators and each and every term in the table must be in accordance with the operator's knowledge and plan. Some KPIs in this table, such as throughput varies both in uplink and downlink and depending on the location, user behaviour etc. So the metrics of the throughput performance indicators are not marked as not KPIs but they are the objectives that need to be measured to monitor the network capacity.

KPI reports on retainability, accessibility, traffic and mobility with handover (HO) attempts were generated based on the previously discussed strategy and in consultation with the operators. The minute details of all the KPIs are not provided in this section. Only a broad KPI analysis is made. The details of each KPI definition and representation are left to the users design depending on the circumstances and the network design and planning.

\section{INTEROPERABILITY TEST AND RESULTS}

Today's networks consist of equipment from different vendors and the interoperability testing between the vendors has become crucial to the successful operation of multi-vendor networks. Interop testing is very important part to be carried out before the network expansion (O.Monkewich, 2010). Network elements from different vendors are tested to identify whether they are confirming to same standards so that better interoperability between them can be achieved. Vendors can implement the standards independently with the assurance that the products will confirm for the interoperability.

The interoperability testing comes with many objectives. The first objective of interoperability testing is to test whether the conformance is met and should not be compromised. The second objective is to test whether the product does the same functionality as specified by the recommendation. The third objective is to test whether the products are built in conformance with the standards specified by ITU because sometimes some vendors don't support all the features specified by the ITU and the features not supported may be required by the operators. In order to test the functionality of the features required by the operators, the interop testing is performed. Each product should be feature tested by designing test cases as agreed by the participating vendors. The capability of LTE/LTE-A to provide higher bandwidth is attracting new customers and the network expansion planning has become very crucial survival of the operators. Hence, the expansion plan should be meticulous and economical. The interoperability testing that was 
carried out focuses on creating a test bed with eNB and MME from different vendors and with the SGW pooling. As a test condition, the UE (user equipment) moves from source eNB to target eNB, while doing so, X2 handover was performed with SGW relocation. In the test bed the IP connectivity exist between source SGW and the source eNB, between the source SGW and target eNB, between the target SGW and target eNB. If the IP connectivity doesn't exist between the target eNB and source SGW, then S1 based handover shall be used instead. From the operators perspective it is good to test the multi vendor scenario because it gives a better business opportunity in terms of cost and benefit. In the tests, the issue in eNB perform signalling gateway (SGW) relocation at path switch request by mobility management entity (MME) was identified. During the interop testing, the emphasis was given to this scenario as the operator was planning to implement a MME manufactured by different vendor.

In Figure 2, the RRC drops can be observed between June 10 to July 13 and the statistics shows the radio resource connection (RRC) drops increased to almost $100 \%$. But the same stats show the normal performance of the network for the period between May 10 to June 10 and the period starting from July 14 to August 14. Figure 3 shows the abnormal radio access bearer (eRAB) release by the MME. The period discussed between June 10 to July 13 shows a heavy eRAB release by MME due to abnormal activity. Similarly in Figure 5, the mobility success and handover attempts show that there is a slump in the success rate for the same period. Further investigation into the issue was performed to analyse the behaviour. In the investigation it was found that during the handover execution phase, the pathswitchrequest is sent from the target eNB to MME and a acknowledgement by means of pathswitchrequest acknowledge message sent from MME to the eNB, However, during this process along with the acknowledge message, MME sends SGW relocation, despite the fact that there is only one SGW configured with the eNB, which had resulted in a failure and hence a RRC release will be triggered affecting the retainability of the system and that can be observed in Figure 2 and Figure 3. This is a very important issue to be tested for the UE trying to perform handover from source eNB to target eNB using X2 interface between the eNB. The X2-based handover failures because of the fake SGW relocation sent by the MME attributed to the reason for the high RRC drops. The X2 handover is performed to handover the UE from serving eNB to a target eNB but within the same MME. The target eNB sends path switch request message to MME to inform that UE has changed the cell, including ECGI of the target cell and EPS bearers. The MME is responsible for selecting the new SGW, since MME knows the service area of the UE to the TA granularity. This interoperability issue was found in the test sites in the network. To solve this issue there are 3 ways to address this problem and they are:

- Software upgrade in the Core is required to not to send SGW relocation when configured with the single $\mathrm{SGW}$ relocation.

- Introduce a patch in the eNodeB to ignore the fake request from the MME

- Introduce a software add in the eNodeB for processing this fake request from the MME

The third option was chosen, since it is future proof and in case if a real request comes from the MME, eNB will start working on the SGW relocation and the eNB software was upgraded to support the feature known as SGW relocation at X2. After introducing this feature, the eNB does not ignore the SGW relocation at path switch request sent by the MME. In Figure 3 starting from July 14, this issue was not observed and the stats has also improved. This issue is a very important fix for the successful sign off of the interoperability stage otherwise if implemented in live network without fixing this issue would have triggered RRC drops all over the network. This issue would not have been observed if the MME and eNB are from the same vendor. But because of the network expansion plan, the MME and eNB from different vendors were tested for interoperability. 


\section{DisCUSSION}

Every network needs the performance indicators to monitor and improve its performance. Key KPIs are vital navigational counters that help the service providers and vendors to understand the network. The right sets of KPIs will always pin-point the right nature of the network performance levels and it helps to shine the light on areas that needs attention. Without KPIs the service providers and vendors appears to be flying blind. But pressing problem in today's complicated and multi layered network is identifying the right set of KPIs and managers are struggling with it. As a consequence to it they end up drowning in data while thirsting for information. The present day network is highly composite in nature and consists of multiple vendors. So in this paper we have proposed the testing of network KPIs in the interop environment to verify the functionality and the correctness of the KPI design by the vendor. This testing helped us to test the robustness of the KPIs and based on the KPIs obtained, the issue in the interop environment was identified. Hence this testing provides a vital proof for the vendor and the service provider to believe in the KPIs design that they together defined and developed.

\section{CONCLuSiON}

There are various testing scenarios are discussed in this paper. First and foremost is the general stability of the network, KPI design for network performance verification and Interoperability between various equipments from various vendors. The stability and availability of the network is the gateway to the success and to achieve this operator's use various KPIs to evaluate the network performance. The next generation mobile networks (NGMN) (NGMN, 2006) alliance has documented the issues that arose in the past network verification approaches. Through this study, the NGMN alliance has recommended the top down approach for the verification of the various network features and its performance. The NGMN study has grouped the network monitoring KPIs into five major groups and they are accessibility, retainability, integrity, availability and mobility. The spotlight of network verification should be aimed on these five major groups and this group in turn is based on the several low level PIs. There are several benefits for the operators if they have proper KPI mechanism to understand about the network and the user experiences and some of them are 1) Helps in understand the network and user experience and they can go to the market fast with new service and coverage. 2) The operators can be fully confident about the network and its performance. 3) Easy benchmarking in the complex multivendor environments 4) it is easy to achieve the targeted efficiency in the network. This KPI mechanism helps the vendors to 1) to focus the resources on network tuning and maximizing the network efficiency 2) network deployment can be done very faster to meet the deadlines 3) to roll out the network and helps them to sustain the competition. Special bonding can be created with the operators to survive the competition. Hence, the KPI analysis is the best form for testing several network related features. In order to sustain and grow in the market, the network expansion has to be supported with planned interoperability tests between equipments from various vendors. This gives the network operators the confidence required to sustain the market. 
International Journal of Wireless \& Mobile Networks (IJWMN) Vol. 7, No. 5, October 2015

\section{REFERENCES}

[1] Web page of LSTI (http://lstiforum.org).

[2] White paper "LTE

deployments",http://br.networks.nokia.com/file/3164/white-paper-lte-performance-initialdeployments

[3] Ericsson, White Paper, 2011, Keeping the Customer Service Experience Promise - How to Meet the Service Assurance Challenge, available at: http://www.ericsson.com/news/110121 wp service assurance $244188811 \mathrm{c}$

[4] ETSI, Technical Specification 132 450, 2009-04, Key Performance Indicators (KPIs) for Evolved Universal Terrestrial Radio Access Network (E-UTRAN ): Definitions (3GPP TS 32.450 Version 8.0.0 Release 8), available at:http://www.etsi.org/deliver/etsi_ts/132400_132499/132450/08.00.00_60/ts_132450v080000p.pdf

[5] 3GPP, Technical Specification 32.450, 2009, Telecommunication Management; Key Performance Indicators (KPIs) for Evolved Universal Terrestrial Radio Access Network (E-UTRAN ): Definitions, available at: http://www.3gpp.org/ftp/Specs/html-info/32450.htm

[6] 3GPP , Technical Report 36.805, 2009, Study on minimization of drive-tests in next generation networks, available at: http://www.3gpp.org/ftp/Specs/html-info/36805.htm

[7] O.Monkewich,"Conformance and Interoperability Testing Tutotial", ITU-T SG 17, available at:https://www.itu.int/dms_pub/itu-t/oth/15/04/T15040000080001PDFE.pdf

[8] NGMN Alliance, White Paper, 2006, Next-Generation Mobile Networks Beyond HSPA \& EV DO, available at: http://www.ngmn.org/uploads/media/Next Generation Mobile Networks Beyond HSPA EV DO web.pdf 


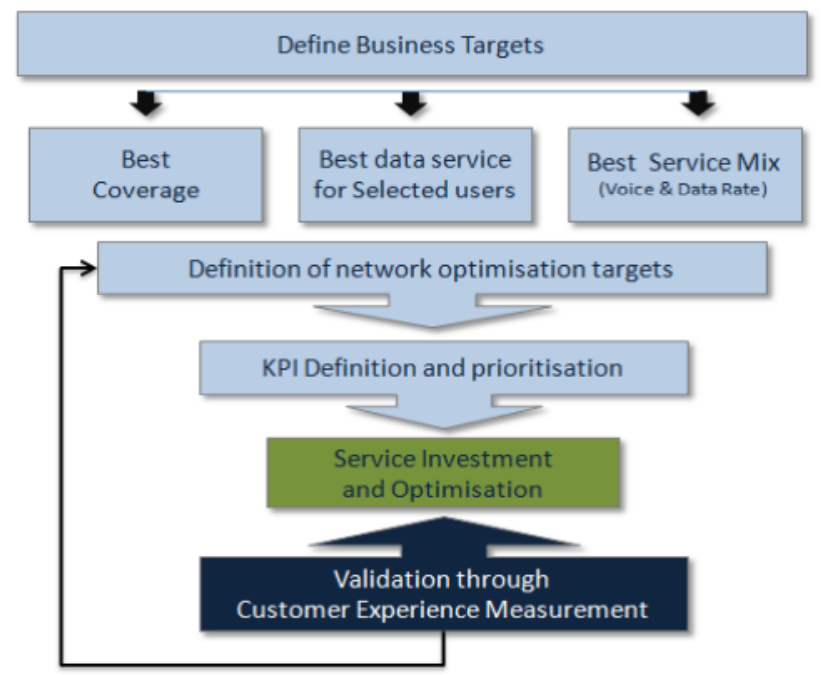

Figure 1. ITU recommended KPI methodology

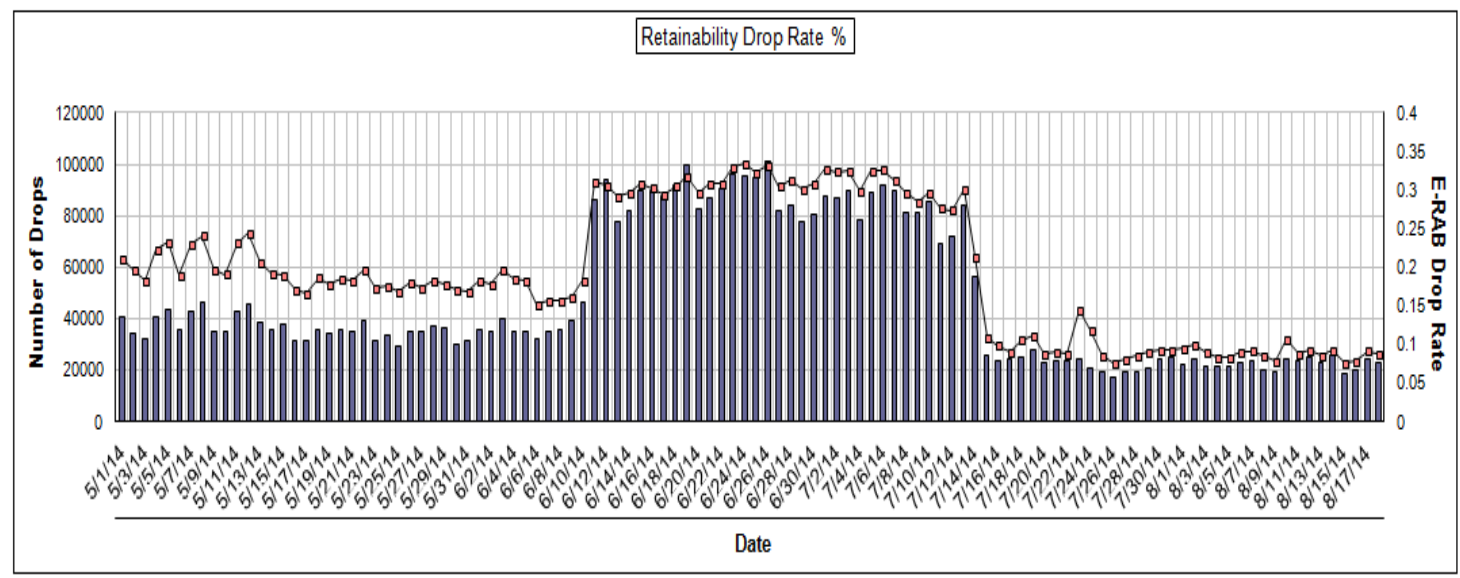

Figure 2. Retainability graph

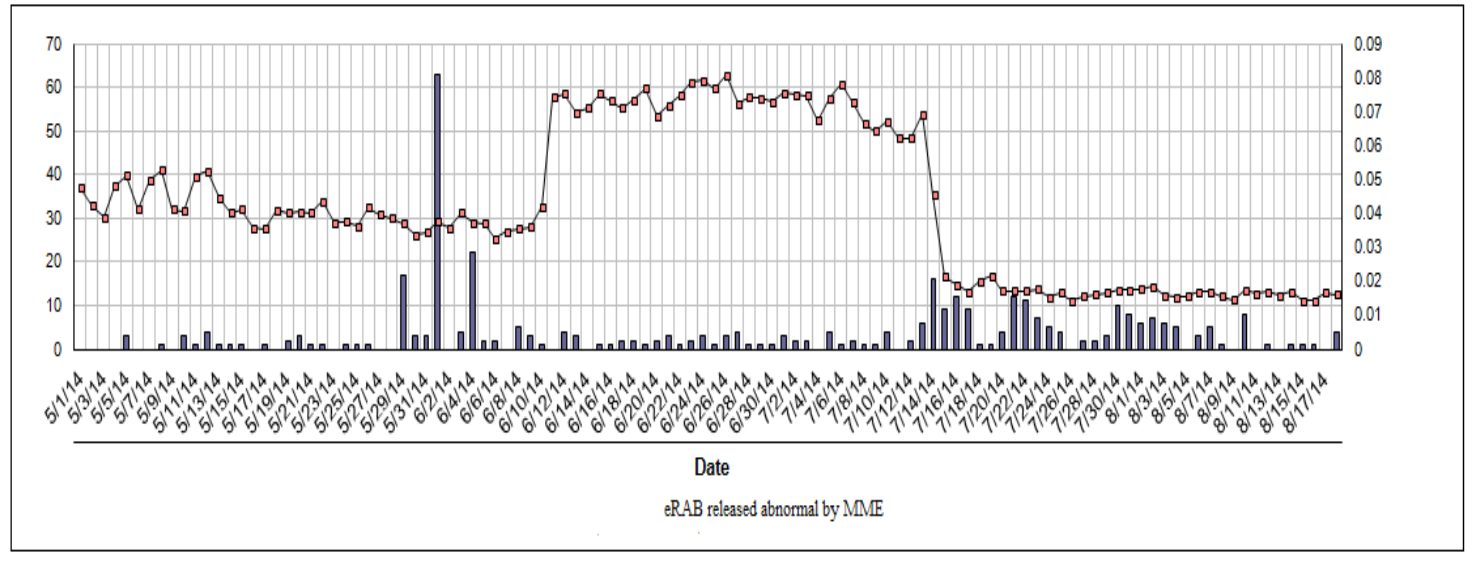

Figure 3. E-Retainability graph 


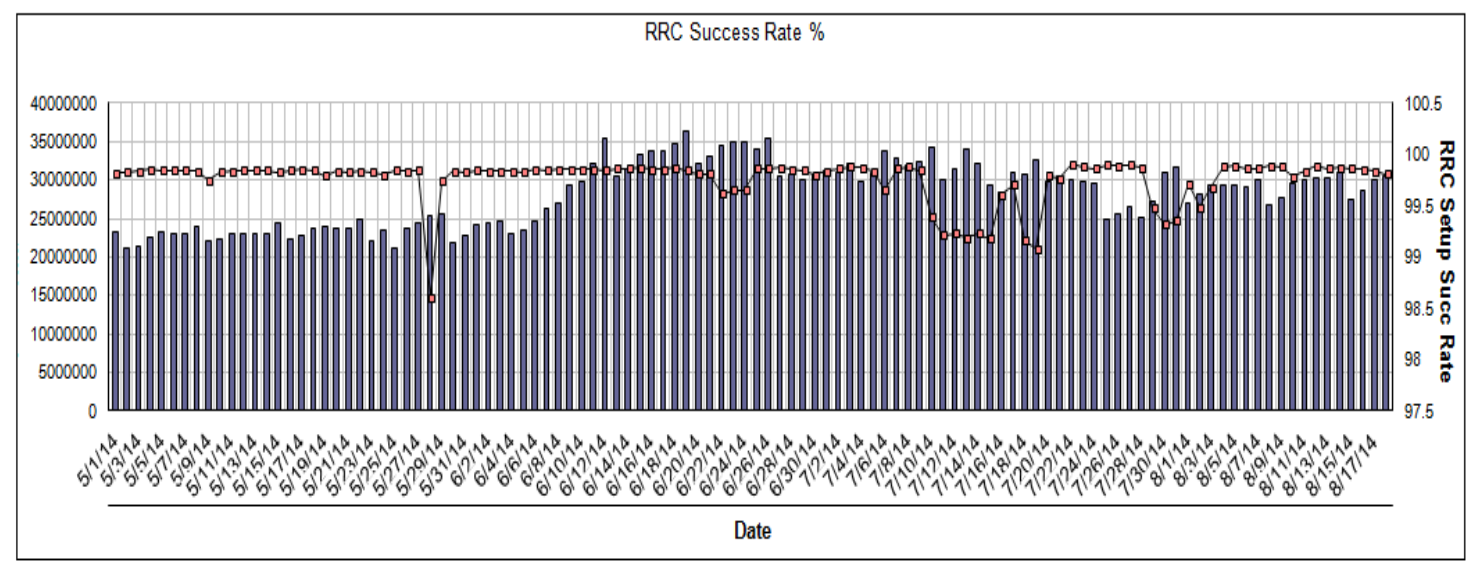

Figure 4. Accessibility graph

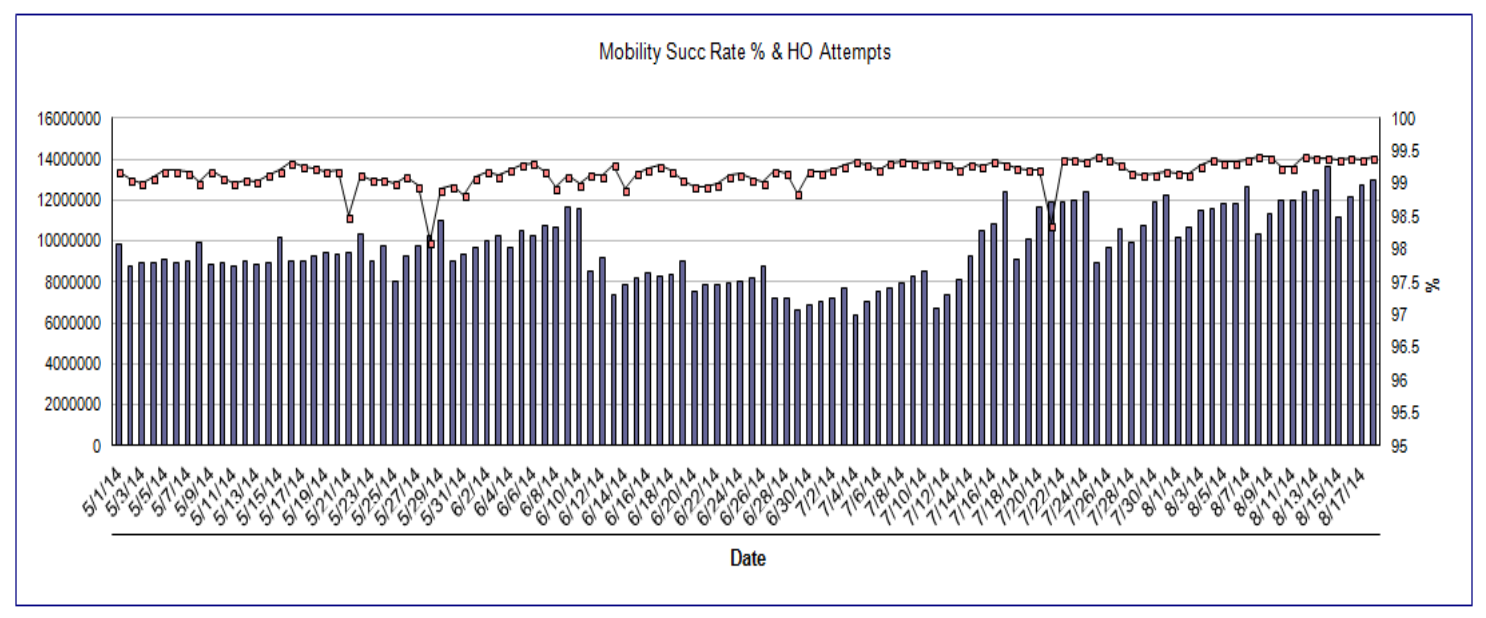

Figure 5. Mobility graph

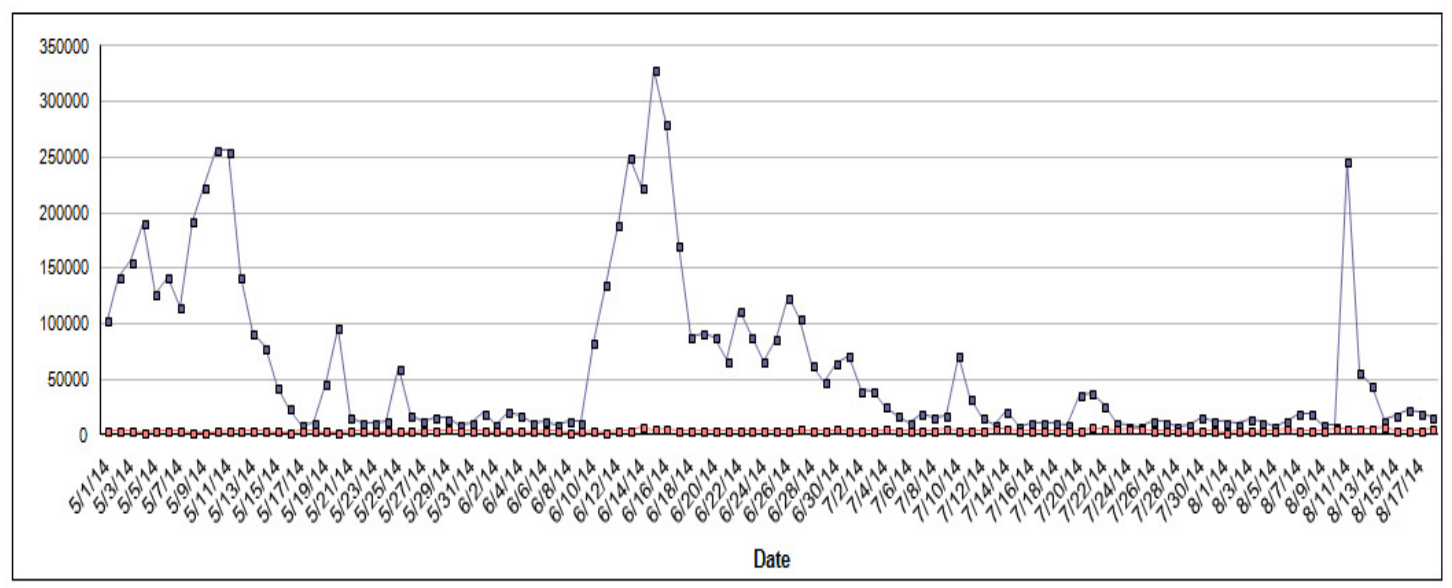

Figure 6. Automatic neighbour relation graph 